\title{
Antisemitismus - Ein blinder Fleck der intersektionalen Geschlechterforschung?
}

\author{
Manuel Mayrl
}

Bereits Ende der 1970er-Jahre wurde innerhalb zeitgenössischer Debatten der feministischen Theoriebildung auch Kritik an antisemitischen Denkmustern laut. Jüdische Feministinnen wie Judith Plaskow und Annette Daum kritisierten die Reproduktion antjüdischer Klischess in der damals formulierten Patriarchatskritik und forderten demgegenüber differenziertere Analysen ein. ${ }^{1}$ Dennoch scheint diese frühe Kritik kaum Eingang in die feministische Reflexion gefunden zu haben. Auch heute noch lassen Feministinnen und Feministen wie auch Geschlechterforscherinnen und Geschlechterforscher wiederholt mit antisemitischen Äußerungen oder Unterstützungsbekundungen für die antisemitische BDS-Kampagne ${ }^{2}$ aufhorchen, darunter Koryphäen wie Judith Butler, Laurie Penny und Angela Davis. Wie kommt es, dass feministische Denkerinnen, die sich zum Teil in der Tradition der Kritischen Theorie verorten, Antisemitismus ausblenden und - „on basis of intersectionality “3 durch eine einseitige Positionierung gegen den israelischen Staat dessen Delegitimierung forcieren?

Mit „intersectionality“ (dt. Intersektionalität) konzeptualisierte Kimberlé Crenshaw die Mechanismen des Zusammenwirkens mehrerer Ungleichheitsdimensionen, jenseits eines simplen Aufaddierens. Konkret untersuchte sie das Zusammenwirken von „race“ und „gender" im Kontext des US-amerikanischen Rechtssystems. Allerdings betonte sie schon früh, das Konzept könne und solle erweitert werden. ${ }^{4}$ Mit Edward Said kann diese Anmerkung Crenshaws verstanden werden als „kritische Einsicht, daß keine Theorie imstande ist, alle Situationen abzudecken, einzugrenzen und vorherzusagen, für die sie nützlich sein könnte“. ${ }^{5}$

1 Charlotte Kohn-Ley/Ilse Korotin (Hrsg.), Der feministische „Sündenfall“? Antisemitische Vorurteile in der Frauenbewegung, Wien 1994, S. 9f.

2 Das Akronym BDS steht für Boycott, Divestment and Sanctions und ist ein „Beispiel für eine einflussreiche antisemitische Kampagne gegen Israel, die strukturell von Kritik unterschieden werden kann." Samuel Salzborn, Israelkritik oder Antisemitismus? Kriterien für eine Unterscheidung, in: Kirche und Israel. Neukirchener Theologische Zeitschrift 28 (2013), Heft 1, S. 5-16, hier S. 11.

${ }^{3}$ Karin Stögner, A Critical Theory of Nationalism, Post-Nationalism and anti-Zionism, Vortrag auf der International Scholars Conference Anti-Zionism, Antisemitism, and the Dynamics of Delegitimization, Bloomington, 2.-6.4.2016, [https://youtu.be/cnDxmsau-dY], eingesehen 11.10.2017.

${ }^{4}$ Kimberlé Crenshaw, Mapping the Margins. Intersectionality, Identity Politics, and Violence Against Women of Color, in: Stanford Law Review 43 (1991), Heft 6, S. 1241-1299, hier Fußnote 9 (S. 1245).

${ }^{5}$ Edward Said, Theorien auf Wanderschaft, in: Ders. (Hrsg.), Die Welt, der Text und der Kritiker, Frankfurt a. M. 1997, S. 263-292, hier S. 283. 
Dieser Beitrag geht von der grundlegenden Offenheit von Crenshaws Konzept aus und schließt sich Saids impliziter Forderung an, theoretische Konzepte an die jeweiligen Kontexte anzupassen. Auf diese Weise soll das konflikthafte Verhältnis zwischen intersektionaler und antisemitismuskritischer Analyse näher beleuchtet werden. Davon ausgehend wird nachgezeichnet, in welchem Kontext sich das ursprüngliche Konzept intersectionality entwickelte und wie es im deutschsprachigen Raum rezipiert wurde. ${ }^{6}$ Dies geschieht auf Grundlage von Edward Saids Anmerkungen zu „traveling theories“ und entsprechend des darin explizit nahegelegten historischen Ansatzes: „[T] is always informed by the past, by past hopes and promises, but also by past horror and injustice. “7 Durch diesen Ansatz soll „[d]as kritische Bewusstsein“ als „ein Wissen um die Unterschiede zwischen den Situationen“8 entwickelt werden, das den Ausgangspunkt für konkrete Konzeptualisierungen von Antisemitismus als Bestandteil von Intersektionalität markieren soll.

\section{Historischer Entstehungskontext in den Vereinigten Staaten}

Der ideengeschichtliche Ursprung von intersectionality wird gemeinhin in den 1960er und 1970er-Jahren in den Vereinigten Staaten von Amerika verortet. Der soziale Kontext dieser Zeit war maßgeblich von ethnisch segregierten Lebenswelten und antirassistischen Bürgerrechtsbewegungen geprägt. Diese Bewegungen kämpften - unter anderem mit ihrem wohl prominentesten Protagonisten Martin Luther King - für ein Ende des bis dahin geltenden juristischen Grundsatzes „separate but equal“", welcher eine Trennung zwischen „weißen“ und „schwarzen“ Amerikanerinnen und Amerikanern legitimierte. Der Civil Rights Act von 1964 und der Voting Rights Act von 1965, als konkrete Erfolge der antirassistischen Bewegungen, beendeten schließlich formal die Segregation und die Benachteiligung schwarzer Personen bei der Wählerregistrierung. Eng mit der Bürgerrechtsbewegung verzahnt war auch die US-amerikanische Studierendenbewegung. Konkrete Überschneidungen zu den Kampagnen und Forderungen der schwarzen Bürgerrechtsbewegung lassen sich etwa am Beispiel des Student Nonviolent Coordinating Committee (SNCC) illustrieren, zu dessen bekanntesten Mitgliedern Angela Davis und Stokely Carmichael zählten. ${ }^{9}$ Anhand des SNCC und dessen zeitweisen Vorsitzenden Stokely Carmichael sollen im Folgenden theoretische Grundlagen,

${ }_{6} \mathrm{Um}$ die unterschiedlichen historischen und gesellschaftlichen Kontexte und die Transformation des Konzepts sprachlich sichtbar zu machen, wird im weiteren Verlauf des Beitrags zwischen intersectionality - für den USamerikanischen Kontext - und Intersektionalität - für den deutschsprachigen Raum - unterschieden. Siehe auch Lucy Chebout, Wo ist Intersectionality in bundesdeutschen Intersektionalitätsdiskursen? - Exzerpte aus dem Reisetagebuch einer Traveling Theory, in: Sandra Smykalla/Dagmar Vinz (Hrsg.), Intersektionalität zwischen Gender und Diversity. Theorien, Methoden und Politiken der Chancengleichheit, Münster 2011, S. 43-57.

${ }^{7}$ Karin Stögner, A Critical Theory.

${ }^{8}$ Edward Said, Theorien auf Wanderschaft, S. 284.

${ }^{9}$ Peniel E. Joseph, Stokely: A Life, New York 2014. 
internationale Vernetzungen und politische Parteinahmen - vor allem in Bezug auf Israel - skizziert werden.

Stokely Carmichael war von 1966 bis Ende 1967 Vorsitzender des SNCC, eine Zeit in der sich seine politischen Positionen zu internationalisieren und zu radikalisieren begannen. Dieser Prozess ging mit einer Zuwendung hin zur Black Power Bewegung und einer Entfremdung von Martin Luther Kings Positionen einher. ${ }^{10} \mathrm{Im}$ Juli 1967 hielt Carmichael in Havanna eine vielbeachtete Rede auf der OLAS Konferenz - einer Nachfolgeorganisation der ein Jahr zuvor gegründeten „Tricontinentalen“, der auch die Palestine Liberation Organisation (PLO) angehörte. Fidel Castro begrüßte ihn dort als „one of America's ,most distinguished' civil rights leaders.“" Wenige Tage zuvor trat Carmichael als Redner bei der Konferenz „Dialectics of Liberation“ in London auf, an der auch Herbert Marcuse und Angela Davis teilnahmen. Sich vom konventionellen Marxismus abgrenzend, identifizierte Carmichael auf dieser Konferenz neben dem Kapitalismus den Rassismus als globales Unterdrückungssystem und forderte eine Solidarisierung mit der sogenannten Dritten Welt: „What we're talking about around the United States today, and I believe around the Third World, is the system of international white supremacy coupled with international capitalism. "12 Entsprechend seiner an Frantz Fanon angelehnten Argumentation, der Befreiungskampf der schwarzen Bevölkerung könne nur in konsequenter Abgrenzung zu - und nicht mit - weißer Unterstützung geführt werden, fasste das SNCC Ende 1966 den Beschluss, sich von seinen weißen Mitgliedern zu trennen. Durch diesen - denkbar knappen - Beschluss wurden auch viele bis dahin aktive jüdische Mitglieder ausgeschlossen, was zu einer beginnenden Entfremdung zwischen vormals vielseitig kooperierenden schwarzen und jüdischen Interessensgruppen führte. ${ }^{13}$

Einen wichtigen theoretischen Bezugspunkt für Carmichael und die der Black Power Bewegung zugewandten Teile der US-amerikanischen Bürgerrechts- und Studierendenbewegungen, stellte Fanons Arbeit zu Gewalt und Kolonialismus dar. Vor dem Hintergrund des Algerienkrieges beschreibt Frantz Fanon in seinem Werk „Die Verdammten dieser Erde" sowohl die Kolonisation als auch die Dekolonisation als notwendig gewaltförmig. Der Status Quo der Kolonisation könne „nur durch die absolute Gewalt in Frage gestellt werden". ${ }^{14}$ Neben einer Affirmation der Gewalt von Kolonisierten, lässt sich in Fanons Arbeit auch eine antimilitaristische Haltung erkennen. Fanon nahm damit eine implizite Unterscheidung zwischen emanzipatorischem Guerillakampf von antikolonialen Befreiungsbewegungen einerseits und negativ

${ }_{10}$ David J. Garrow, Bearing the Cross. Martin Luther King Jr. and the Southern Christian Leadership Conference, New York 1986.

${ }^{11}$ Joseph, Stokely, S. 134.

12 Zit. n. ebd., S. 132.

${ }_{13}$ Jewish Virtual Library, Modern Jewish History: The New Left, o. D., [http://www.jewishvirtuallibrary.org/the-newleft], eingesehen 7.10.2017.

${ }^{14}$ Frantz Fanon, Die Verdammten dieser Erde, Sonderausgabe, Frankfurt a. M. 2008, S. 29. 
konnotierten staatlichem Militarismus andererseits vor. Diese Unterscheidung des Partikularen - der emanzipatorischen Gewalt der Kolonialisierten - gegenüber dem Universalen - dem Unterdrückungsregime und dessen Militarismus - lässt sich auch bei Angela Davis wiederfinden. Sie beschreibt Israel als abstrakten und künstlichen Staat, der in diesem Sinne für das Universale und eine weiße, männliche Herrschaft einstehe. Demgegenüber stellt sie die arabischen Gemeinschaften, die noch authentisch und verwurzelt wären. ${ }^{15}$ Nicht nur wird hierbei das dialektische Verhältnis zwischen dem Besonderen und dem Allgemeinen ,zugunsten antikolonialer ,Eindeutigkeit"“16 verkannt. Ebenso greifen die impliziten wie expliziten Dichotomien wurzellos/verwurzelt, konkret (authentisch)/abstrakt (künstlich), Gemeinschaft/Gesellschaft klassische Strukturelemente des modernen Antisemitismus auf. ${ }^{17}$

Im Laufe der Zeit verschärfte sich Carmichaels antisemitische Rhetorik und gipfelte 1985 in der Aussage: „The only good Zionist is a dead Zionist." 18 Hier wird ein konkreter Konfliktpunkt zu Martin Luther King deutlich, der bereits 1968 den sich ausbreitenden Antizionismus in der amerikanischen Linken als Antisemitismus entlarvte: „When people criticize Zionists, they mean Jews. You're talking anti-Semitism. “19

Die „Studentenbewegung selber war [...] mit ihren Themen und ihren Herangehensweisen ein wichtiges Fundament für die neue Frauenbewegung " ${ }^{20}$ und folglich auch für die konzeptionelle Entwicklung von intersectionality. ${ }^{21}$ Neben die antirassistische Bürgerrechtsbewegung und die antiimperialistische Studierendenbewegung trat eine an Geschlechtergerechtigkeit orientierte Frauenbewegung. Die im anglo-amerikanischen Raum bis heute dominierende Konzeptualisierung von intersectionality (race, class, gender) ist das Ergebnis eines historischen Integrationsprozesses dieser sozialen Bewegungen im Rahmen eines Analysekonzepts. ${ }^{22}$

Dass die theoretischen Auseinandersetzungen der beiden zuvor skizzierten Bewegungen auch an der inhaltlichen Ausrichtung der Frauenbewegung nicht spurlos vorübergegangen sind, wurde spätestens bei der ersten UN-Weltfrauenkonferenz 1975

15 Karin Stögner, New Challenges in Feminism: Intersectionality, Critical Theory, and Anti-Zionism, in: Alvin H. Rosenfeld (Hrsg.), Anti-Zionism and Antisemitism. The Dynamics of Delegitimation. Bloomington 2018, S. 84-112. ${ }^{16}$ Martin Kloke, Antizionismus und Antisemitismus als Weltanschauung? Tendenzen im deutschen Linksradikalismus und -extremismus, in: Bundesministerium des Inneren (Hrsg.), Texte zur inneren Sicherheit. Extremismus in Deutschland. Erscheinungsformen und aktuelle Bestandsaufnahmen, Berlin 2004, S. 163-196, hier S. 170.

17 Claudia Globisch, Radikaler Antisemitismus. Inklusions- und Exklusionssemantiken von links und rechts in Deutschland, Wiesbaden 2013.

${ }^{18}$ Henry Louis Gates Jr./Evelyn Brooks Higginbotham, African American Lives, Oxford-New York 2004, S. 142.

19 Zit. n. Seymour Martin Lipset, The Socialism of Fools. The Left, the Jews, and Israel, New York 1969, S. 7.

${ }^{20}$ Frigga Haug, Perspektiven eines sozialistischen Feminismus. 20 Jahre Frauenbewegung in Westdeutschland und West-Berlin, in: Autonome Frauenbewegung (Hrsg.), Westeuropa (Frauenbewegungen in der Welt 1), Berlin 1988, S. 25-52, hier S. 27.

${ }^{21}$ Sara Salem, Intersectionality and its discontents: Intersectionality as traveling theory, in: European Journal of Women's Studies (2016), S. 1-16, hier S. 10.

${ }^{22}$ Eve Mitchell, I am a woman and a human. A Marxist feminist critique of intersectionality theory, 12.9.2013, [https://libcom.org/library/i-am-woman-human-marxist-feminist-critique-intersectionality-theory-eve-mitchell], eingesehen 9.10.2017. 
sichtbar. Durch eine Mehrheit aus kommunistischen und arabischen Staaten wurde eine Deklaration verabschiedet, die sich gegen jede Form der Unterdrückung richtet und Frauen als ,natural allies in the struggle against [...] colonialism, neocolonialism, Zionism, racial discrimination and apartheid" deklarierte. ${ }^{23}$ Dieser Passus, der später informell als „Zionism is racism resolution“ bezeichnet wurde, sollte kurze Zeit später auch von der UN-Generalversammlung in der Resolution 3379 verabschiedet werden. Bemerkenswerterweise wurde der Vorstoß von Delegierten aus Neuseeland, Sexismus ebenfalls der Aufzählung hinzuzufügen, von eben dieser Mehrheit abgelehnt. ${ }^{24}$ Gerade in Bezug auf die Weltfrauenkonferenz gilt es anzumerken, dass die Frauen, die an den offiziellen Treffen als Repräsentantinnen ihrer Regierungen teilnahmen, letztlich unter der Kontrolle männlicher Politiker in ihren Heimatländern standen. ${ }^{25}$ Es ist daher wenig überraschend, dass auch innerhalb der US-Delegation nicht alle Feministinnen mit der Gegenstimme zur „Zionism is racism resolution“ einverstanden waren. ${ }^{26}$ Vor allem Women of Color identifizierten sich zum Teil mit den Palästinenserinnen und Palästinensern, mit der Begründung, diese seien ,people of color struggling for the liberation of their homeland." 27

Nicht nur in offiziellen Delegationen wurden zu diesem Zeitpunkt die Stimmen von Women of Color überhört. Auch innerhalb der Bürgerrechtsbewegung gerieten die spezifischen Bedürfnisse und Erfahrungen schwarzer Frauen zugunsten einer möglichst großen und homogen auftretenden Interessengruppe häufig in den Hintergrund. Auf der anderen Seite fühlten sich viele schwarze Frauen auch von der damals ebenfalls an Einfluss gewinnenden zweiten Welle des Feminismus, der vorwiegend von weißen Mittelschichtsfrauen getragen wurde und in seinen Forderungen an deren Lebensrealitäten ansetzte, nicht entsprechend repräsentiert. Um sich der jeweils einseitigen Vereinnahmung einer dieser beiden sozialen Bewegungen zu entziehen, gingen schwarze Feministinnen dazu über, eigene Organisationen zu gründen. ${ }^{28}$ Das Combahee River Collective gilt als eine der bekanntesten und einflussreichsten Gruppierungen jener Zeit. Es konstituierte sich 1974 in Boston und veröffentlichte drei Jahre später das vielrezipierte „Combahee River Collective Statement“. Darin formulierte das Kollektiv seinen Anspruch „racial, sexual, heterosexual, and class oppression“ zu bekämpfen, da es davon ausging, dass , [t] $[$ he synthesis of these oppressions creates the

${ }^{23}$ UN Documents, Declaration of Mexico on the Equality of Women and their Contribution to Development and Peace, 2.7.1975, [http://www.un-documents.net/mex-dec.htm], eingesehen 8.10.2017.

${ }^{24}$ Betty Friedan, It changed my life: Writings on the Women's Movement, Cambridge, Massachusetts 1998.

${ }^{25}$ Kristen Ghodsee, Revisiting the United Nations decade for women: Brief reflections on feminism, capitalism and Cold War politics in the early years of the international women's movement, in: Women's Studies International Forum 33 (2010), S. 3-12, hier S. 5.

${ }^{26}$ Hannah Milstein, The United Nation Women's Decade and Jewish Feminist Identity, in: Ex Post Facto 25 (2016), S. 199-222.

${ }_{27}$ Barbara Smith, The Truth That Never Hurts. Writings on Race, Gender, and Freedom, New Brunswick (N.J.) 1985, S. 149.

${ }^{28}$ Patricia Hill Collins/Sirma Bilge, Intersectionality, Cambridge 2016. 
conditions of our lives“. ${ }^{29}$ Obwohl die Gruppe den Begriff intersectionality noch nicht verwendete, ist das später von Kimberlé Crenshaw ausgearbeitete Konzept bereits deutlich erkennbar.

Ende der 1980er-Jahre führte die Rechtswissenschaftlerin Kimberlé Crenshaw schließlich den Begriff intersectionality ein. ${ }^{30}$ Sie konzeptualisierte damit einen juristischen Fall, in dem eine Diskriminierung schwarzer Frauen aufgrund ihrer spezifischen Situation von der US-amerikanischen Rechtsprechung nicht erfasst werden konnte. Der Gesetzeslage lag zu diesem Zeitpunkt ein eindimensionaler Ordnungsrahmen zugrunde, basierend auf der Annahme, dass eine Person entweder aufgrund der Rasse (race) oder aufgrund des Geschlechts (gender) diskriminiert werden könne. Eine Diskriminierung aufgrund der Gleichzeitigkeit dieser beiden Kategorien war dabei konzeptionell nicht vorgesehen. Intersectionality ist eine komplexe Metapher, die darauf abzielt, genau dieses Zusammenwirken von zwei oder mehreren Unterdrückungskategorien sichtbar $\mathrm{zu}$ machen und $\mathrm{zu}$ benennen. Das Konzept baut auf der grundlegenden Erkenntnis auf, dass soziale Ungleichheit und lebensweltliche Erfahrungen durch multiple und ineinander verschränkte Achsen sozialer Einteilung geprägt sind, die zusammenwirken und sich gegenseitig beeinflussen. ${ }^{31}$

Crenshaws Überlegungen zur intersectionality besonders im Kontext US-amerikanischer Rechtsprechung, fand auch andernorts großen Zuspruch. Sara Salem spricht gar von einer neuen Phase in der Geschlechterforschung, die damit eingeleitet wurde. ${ }^{32}$ Die Übernahme und Übersetzung von intersectionality in anderen fachlichen und regionalen Gebieten wird häufig als Fall einer ,traveling theory“ genannt. ${ }^{33}$ Damit wird in Anschluss an Edward Saids Ausführungen theoretisiert, dass sich Theorien, Konzepte und Ideen im Zuge einer regionalen, zeitlichen oder fachlichen Aneignung verändern können. Said geht davon aus, dass Ideen und Theorien wandern, „,von Mensch zu Mensch, von Situation zu Situation, von einer Epoche zur anderen“.34 Im Zuge dieser Wanderung können sich diese Ideen verändern, denn der Übergang geschieht nie reibungslos. Häufig diskutiert wurde die Veränderung von intersectionality im Zuge der Übernahme im deutschsprachigen Kontext etwa in Bezug auf die angemessene Bezeichnung der Kategorie „race“.

29 Text abrufbar über die Webseite der Yale University: The Combahee River Collective Statement, o. D. [https://americanstudies.yale.edu/sites/default/files/files/Keyword\%20Coalition_Readings.pdf], eingesehen 2.10.2017.

30 Kimberlé Crenshaw, Demarginalizing the Intersection of Race and Sex: A Black Feminist Critique of Antidiscrimination Doctrine, Feminist Theory and Antiracist Politics, in: University of Chicago Legal Forum 1 (1989), S. 139-167.

31 Collins/Bilge, Intersectionality, S. 2.

32 Salem, Intersectionality.

${ }^{33}$ Gudrun-Axeli Knapp, Race, Class, Gender. Reclaiming Baggage in Fast Traveling Theories, in: European Journal of Women's Studies 12 (2005), Heft 3, S. 249-265; Chebout, Wo ist Intersectionality; Salem, Intersectionality.

${ }^{34}$ Said, Theorien, S. 263. 
Nachdem bislang die Gegebenheiten am Ursprungsort des Konzeptes behandelt wurden, wird im Folgenden nun - entsprechend Saids Anspruch eines historischen Ansatzes zur Erklärung der Genese und Transformation von Konzepten - der geschichtliche Kontext im deutschsprachigen Raum skizziert.

\section{Historischer Kontext im deutschsprachigen Raum}

Auschwitz - als Symbol für die nationalsozialistische Judenvernichtung - markiert eine „Epochenscheide der Antisemitismusgeschichte“. ${ }^{35}$ Vor allem die Nachfolgestaaten des Nationalsozialismus sahen sich mit der Notwendigkeit konfrontiert, den Antisemitismus öffentlich zu ächten, sich von ihm zu distanzieren und ihr Verhältnis zu Jüdinnen und Juden neu zu ordnen. ${ }^{36}$ Dass die Tabuisierung in der politischen Kultur nicht auch zu einem radikalen Wandel tiefsitzender Einstellungsmuster führte, belegt unter anderem das 1954 veröffentlichte „Gruppenexperiment“ des kurz zuvor wiedereröffneten Frankfurter Instituts für Sozialforschung. Die Studie legte den insbesondere unter Akademikerinnen und Akademikern immer noch weit verbreiteten Antisemitismus offen und folgerte, „daß zwar die nationalsozialistische Ideologie als einheitlich organisierter Zusammenhang nicht mehr existiert, [...] daß aber zahlreiche Einzelelemente des faschistischen Denkens [...] noch gegenwärtig sind “. ${ }^{37}$ Vor dem Hintergrund solcher Befunde ist es wohl kein Zufall, dass die Vertreterinnen und Vertreter der Kritischen Theorie zu den vehementesten Unterstützerinnen und Unterstützern eines souveränen jüdischen Staates zählten. ${ }^{38}$

Die Erfahrung der Shoah und der anhaltende Antisemitismus in weiten Teilen Europas bestärkten Sympathien in der öffentlichen Wahrnehmung für die Notwendigkeit dieses Staates. Nach der Staatsgründung konnte ein Rückgang des Antisemitismus in westeuropäischen Ländern verzeichnet werden. Bedingt unter anderem durch den Eichmann-Prozess, der Veröffentlichung des Tagebuchs der Anne Frank und historischen Forschungen, rückten - vor allem in der Bundesrepublik Deutschland - die eigene Mitschuld und die jüdischen Opfer der Shoah langsam in die Medienberichterstattung und ins öffentliche Bewusstsein. ${ }^{39}$ Zentrales Moment der öffentlichen Aufarbeitung in Deutschland stellte die 68er Bewegung und ihre Kritik an der Nichtauseinandersetzung der postnazistischen Gesellschaften mit den NSVerbrechen dar.

${ }^{35}$ Herbert A. Strauss, Der Holocaust als Epochenscheide der Antisemitismusgeschichte: historische Diskontinuitäten, in: Werner Bergmann/Rainer Erb (Hrsg.), Antisemitismus in der politischen Kultur nach 1945, Opladen 1990, S. 38-56.

${ }^{36}$ Globisch, Radikaler Antisemitismus.

${ }^{37}$ Friedrich Pollock, Gruppenexperiment. Ein Studienbericht, Frankfurt a. M. 1955, S. 397.

38 Stögner, A Critical Theory.

39 Werner Bergmann, Geschichte des Antisemitismus, Beck 42010. 
Verstärkt durch das anfängliche sozialistische Selbstverständnis des jüdischen Staates, solidarisierte sich ein Teil der Linken (auch der Studierendenbewegung) mit Israel, dessen „Existenz der stärkste Beleg für die Verbrechen von Nationalsozialismus und Faschismus war" ${ }^{\prime 40}$. Die von linken Studierenden in Israel gesetzte Hoffnung wurde mit dessen zunehmender Integration in die kapitalistische Weltwirtschaft allerdings enttäuscht und die Sympathien begannen zu schwinden. ${ }^{41}$ Einen „Vertrauensverlust“ hatte Israel in Teilen der Linken schon Mitte der 1950er-Jahre erlitten, als sich der junge Staat an der Seite der Kolonialmächte Großbritannien und Frankreich am Suezkrieg beteiligte.

In dieser Phase internationaler linker Theoriebildung, in der „sozialistische Befreiungsbewegungen" als antiimperialistische Speerspitzen einer globalen Revolution angesehen wurden, unternahm Israel Anfang Juni 1967 einen erfolgreichen Präventivschlag gegen die militärischen Provokationen und Drohgebärden des - von der Sowjetunion unterstützten und ausgerüsteten - ägyptischen Militärs. ${ }^{42}$ Durch seine Allianz mit den Kolonialmächten Frankreich und Großbritannien im Suezkrieg und der Solidarisierung der USA im Zuge des Sechstagekrieges konnte Israel im dichotomen Denken der 68er nicht länger als unterdrücktes und subalternes Kollektiv firmieren. Israel wurde ab diesem Zeitpunkt als imperialistischer Aggressor identifiziert, mit sehr realen Folgen auch für die noch im Westen lebenden Jüdinnen und Juden. Ganz im Sinne der ,politische[n] und organisatorische[n] Zusammenarbeit zwischen den revolutionären Befreiungsbewegungen in der Dritten Welt und den Widerstandsbewegungen in den USA und in den westeuropäischen Ländern" wurden unter anderem Vorträge gestört und jüdische Einrichtungen angegriffen. ${ }^{43}$

Dieser Wandel in der Einstellung gegenüber Israel hatte auch Auswirkungen in der neuen Frauenbewegung. Denn obwohl diese nicht unmittelbar aus den 68ern heraus entstand, können personelle und normativ-ideelle Überschneidungen und Rückgriffe auf bewährte Aktions- und Organisationsformen ausgemacht werden. ${ }^{44}$

Noch während der Hochphase der 68er war das Verhältnis der eigenen Organisationen zu den darin tätigen Frauen widersprüchlich. Das stets betonte Emanzipationsinteresse reduzierte die Interessen der Frauen auf einen Nebenwiderspruch und reproduzierte beständig tradierte Geschlechterrollen. Frauen konnten sich in Debatten kaum Gehör verschaffen und waren vor allem für administrative Aufgaben - wie Kaffee kochen,

40 Anton Pelinka, Israel. Ausnahme- oder Normalstaat, Wien 2015, S. 151.

41 Ebd.

42 Timo Stein, Zwischen Antisemitismus und Israelkritik. Antizionismus in der deutschen Linken, Wiesbaden 2011.

43 Infopartisan, Die Schlußerklärung der Internationalen Vietnam-Konferenz, o. D., [http://www.infopartisan.net/archive/1967/266763.html], eingesehen 5.10.2017.

44 Kristina Schulz, Wende im Geschlechterverhältnis? Feminismus und Frauenbewegung, in: Jens Kastner/David Mayer (Hrsg.), Weltwende 1968? Ein Jahr aus globalgeschichtlicher Perspektive (Globalgeschichte und Entwicklungspolitik 7), Wien 2008, S. 38-53, hier S. 49. 
Flugblätter abtippen und dergleichen - zuständig. Um die Interessen der Frauen vermehrt in den Fokus zu rücken, bildeten sich deshalb eigene Frauenorganisationen. ${ }^{45}$ Die europäische Frauenbewegung der 1970er-Jahre war maßgeblich durch den USamerikanischen Diskurs beeinflusst. Schlüsselkonzepte aus den USA, wie die Patriarchatsanalysen von Kate Millett, haben die „kognitive Orientierung des europäischen Feminismus“ wesentlich geprägt. ${ }^{46}$ Interdependenztheorien, ähnlich zur Intersektionalität, waren allerdings auch im deutschsprachigen Raum nichts gänzlich Neues. Katharina Walgenbach führt mit Clara Zetkin und Mathilde Vaerting zwei einflussreiche, deutschsprachige Denkerinnen an, die sich bereits in den 1920er-Jahren mit den Zusammenhängen von Klasse und Geschlecht auseinandersetzten. ${ }^{47}$ Des Weiteren skizziert sie die Perspektive jüdischer Frauen auf den feministischen Diskurs im deutschsprachigen Raum. Cathy Gelbin beschreibt diesbezüglich sowohl die gemeinsame „Erfahrung einer diskursiven Ausgrenzung auch im Kontext der deutschen Frauenbewegung" migrantischer, schwarzer und jüdischer Frauen, als auch die Konflikte unter eben diesen Gruppen. ${ }^{48}$ So weist sie auch darauf hin, dass jüdische Themen verworfen wurden, da sie im multikulturalistischen Diskurs als $\mathrm{zu}$ dominant wahrgenommen wurden. Jüdinnen wurden als „weiß“ markiert und damit als Teil der Mehrheitsgesellschaft ausgemacht. Diese scheinbare nicht-Subalternität hing nach Gelbin mit der sichtbaren Repräsentation jüdischer Themenbereiche in Form einer aktiven Erinnerungskultur zusammen, während paradoxerweise doch gerade diese Form der Repräsentation zu einer ,,verdrängte[n] Wahrnehmung einer jüdischen Gegenwart im Nachkriegsdeutschland“ führte. ${ }^{49}$

Maria Baader beschrieb in den 1990er-Jahren ihr „Unbehagen am Nachkriegsdeutschland“, das auch mit dem Fehlen jüdischer Feministinnen in der Öffentlichkeit zusammenhing. Dieser Mangel führte 1984 in Berlin auch zur Konstituierung des sogenannten „Schabbeskreis“. ${ }^{50}$ Der Kreis bestand aus einem Zusammenschluss jüdischer und nicht-jüdischer, migrantischer und nicht-migrantischer Frauen, die sich „für jüdische Themen und gegen Antisemitismus engagieren wollten“. 51 Ein Anliegen, das in der deutschen Frauenbewegung auch auf Widerstand stieß. Als (vermeintliche)

${ }^{45}$ Haug, Perspektiven, S. 27.

46 Schulz, Wende, S. 41.

${ }^{47}$ Katharina Walgenbach, Gender als interdependente Kategorie, in: Katharina Walgenbach u. a. (Hrsg.), Gender als interdependente Kategorie. Neue Perspektiven auf Intersektionalität, Diversity und Homogenität, Opladen 2007, S. 23-64.

${ }^{48}$ Cathy S. Gelbin, Die jüdische Thematik im (multi)kulturellen Diskurs der Bundesrepublik, in: Cathy S. Gelbin u. a. (Hrsg.), AufBrüche. Kulturelle Produktion von Migrantinnen, Schwarzen und jüdischen Frauen in Deutschland, Taunus 1999, S. 87-111, hier S. 87f.

49 Ebd., S. 91.

${ }^{50}$ Maria Baader, Zum Abschied. Über den Versuch als jüdische Feministin in der Berliner Frauenszene einen Platz zu finden, in: Ika Hügel u. a. (Hrsg.), Entfernte Verbindungen. Rassismus, Antisemitismus, Klassenunterdrückung, Berlin 21999, S. 82-94.

${ }^{51}$ Gelbin, Die jüdische Thematik, S. 97. 
Jüdinnen, so berichtet Baader, reichte „[d]er Name unserer Gruppe im Programmheft“52, um Anfeindungen ausgesetzt zu werden und zudem als Projektionsfläche für den Hass gegen den jüdischen Staat zu dienen: „Wollten wir etwa die Berührungspunkte zwischen neuen Mütterlichkeitsidealen und antisemitischen Weltbildern aufzeigen, so wurden wir zu unserer Haltung bezüglich der israelischen Politik in den besetzten Gebieten befragt. “53 Zumindest in dieser Zeit konnten jüdische Aktivistinnen somit ausschließlich als Frau, ohne eine betont jüdische Identität, in der Frauenbewegung aktiv sein oder aber sie drohten - im anderen Fall - als Jüdin zur Projektionsfläche eines israelbezogenen Antisemitismus zu werden. ${ }^{54}$ Diese Konflikte führten schließlich zu einem Rückzug jüdischer Feministinnen aus multikulturalistischen Zusammenhängen. ${ }^{55}$ Charlotte KohnLey brachte dies folgendermaßen auf den Punkt: „Es ist für eine jüdische Frau unmöglich, sich ohne Selbstverleugnung feministischen Gruppierungen in Deutschland und Österreich anzuschließen“. ${ }^{6}$ Die bereits zuvor wenig erfolgreichen Bemühungen „eine konkrete Analyse des Antisemitismus als spezifische Unterdrückungsform einzufordern" 57 und in Folge die Betrachtung ihrer Intersektion mit anderen Herrschaftsverhältnissen, kamen damit zum Erliegen.

\section{(Dis)Kontinuität von Antisemitismus in postnazistischen Gesellschaften}

„Nicht einmal der Bruch der Zivilisation, die Realisierung des Antisemitismus in der nationalsozialistischen Judenvernichtung konnte - nicht im Land der Täter - die Kontinuität des Antisemitismus durchbrechen. Der gegenwärtige Antisemitismus ist trotz mannigfaltiger Modifikationen strukturell gleich dem Antisemitismus, der in die Vernichtung der Juden führte“, stellte Klaus Holz Mitte der 1990er fest. ${ }^{58}$ Dass Antisemitismus im deutschsprachigen Raum nach wie vor alltägliche Wirklichkeit ist, das bestätigen unter anderem die jährlich erscheinenden Berichte der Recherche- und Informationsstelle Antisemitismus (RIAS) in Berlin. RIAS registrierte im Jahr 2017 alleine in Berlin 947 antisemitische Vorfälle. ${ }^{59}$ Auch die öffentliche Debatte um die Verbreitung von Antisemitismus im deutschsprachigen Rap, ausgelöst durch die

52 Baader, Zum Abschied, S. 85.

${ }^{53}$ Ebd., S. 86.

54 Ebd.

55 Gelbin, Die jüdische Thematik.

${ }^{56}$ Charlotte Kohn-Ley, Antisemitische Mütter - Antizionistische Töchter?, in: Charlotte Kohn-Ley/Ilse Korotin (Hrsg.), Der feministische „Sündenfall“? Antisemitische Vorurteile in der Frauenbewegung, Wien 1994, S. 209-230, hier S. 229.

57 Gelbin, Die jüdische Thematik, S. 98

58 Klaus Holz, Antisemitismus als Ideologie? Zur Struktur des antisemitischen Selbst- und Gesellschaftsbildes, in: Hansjörg Bay/Christoph Hamann (Hrsg.), Ideologie nach ihrem ,Ende‘. Gesellschaftskritik zwischen Marxismus und Postmoderne, Opladen 1995, S. 149-164, hier S. 151.

${ }_{59}$ Recherche- und Informationsstelle Antisemitismus Berlin, Antisemitische Vorfälle 2017, 2018, [https://reportantisemitism.de/media/bericht-antisemitischer-vorfaelle-2017.pdf], eingesehen 7.5.2019. 
Verleihung des Musikpreises Echo an die Rapper Kollegah und Farid Bang im April 2018, führt vor Augen, dass Antisemitismus weder überwunden, noch das alleinige Problem von isolierten rechtsextremen Gruppierungen ist. Auch im politisch linken Spektrum ist Antisemitismus, etwa in Form einer regressiven Kapitalismuskritik, keineswegs erledigt. ${ }^{60}$ Vielmehr lassen sich antisemitische Einstellungsmuster, wie die Leipziger Mitte-Studien belegen, quer durch alle gesellschaftlichen Schichten und Milieus beobachten. ${ }^{61}$ An öffentlicher Aufmerksam nimmt neuerdings, im Windschatten des langen Sommers der Migration, die Debatte um die Spezifik und Ausprägung eines womöglich „importierten“ Antisemitismus zu. Dieser vermeintliche „neue Antisemitismus der Anderen“62 stellt eine Engführung der Debatte auf den Antisemitismus von Migrantinnen und Migranten dar und ist als Ausdruck einer Externalisierungs-strategie zu verstehen.

\section{Intersektion mit anderen gesellschaftlichen Machtverhältnissen}

Im Folgenden soll ein Überblick über die - in ihrer Anzahl überschaubaren - Ansätze zur Integration von Antisemitismus in intersektionale Betrachtungen gegeben werden. Die bislang intensivste Auseinandersetzung hierzu fand in der Analyse des Zusammenspiels mit Geschlecht statt. Beginnend mit der Auseinandersetzung um die (Mit-)Täterinnenschaft von Frauen während des Nationalsozialismus wurden unter anderem die Spezifika von Frauen als Antisemitinnen ${ }^{63}$, Frauen als „Opfer“ aufgrund ihres Jüdisch-seins ${ }^{64}$ und geschlechterreflektierte Formen der Antisemitismusprävention $^{65}$ in den Blick genommen. „Die verschiedenen historischen und gegenwärtigen Erscheinungsformen des Antisemitismus“, argumentiert Heike Radvan, „waren und sind immer auch vergeschlechtlicht." 66

Eine weitere Überkreuzung, die eher implizit thematisiert wird, ist jene mit kapitalistischen Ausbeutungsverhältnissen. Dieses Verhältnis scheint von Grund auf intersektional zu sein: „Der bürgerliche Antisemitismus hat einen spezifischen ökonomischen Grund: die Verkleidung der Herrschaft in Produktion“. ${ }^{67}$ Jüdinnen und

60 Globisch, Radikaler Antisemitismus.

${ }^{61}$ Oliver Decker/Johannes Kiess/Elmar Brähler, Die enthemmte Mitte. Autoritäre und rechtsextreme Einstellungen in Deutschland, Gießen ${ }^{2} 2016$.

${ }^{62}$ Sina Arnold, Der neue Antisemitismus der Anderen? Islam, Migration und Flucht, in: Christian Heilbronn/Doron Rabinovici/Nathan Sznaider, Neuer Antisemitismus? Fortsetzung einer globalen Debatte, Berlin 2019, S. 128-158.

${ }^{63}$ Ljiljana Radonić, Die friedfertige Antisemitin? Kritische Theorie über Geschlechterverhältnisse und Antisemitismus, Frankfurt a. M. 2004.

${ }^{64}$ Jessica Jacobi/Lwanga Gotlinde Magiriba, Was „sie“ schon immer über Antisemitismus wissen wollten, aber nie zu denken wagten, in: Beiträge zur feministischen Theorie und Praxis (1990), Heft 211, S. 95-105.

65 Heike Radvan, Antisemitismus und Geschlecht. Überlegungen hinsichtlich einer geschlechterreflektierten Prävention, in: Jahrbuch für Antisemitismusforschung 22 (2013), S. 123-142.

${ }^{66}$ Ebd., S. 126.

${ }^{67}$ Max Horkheimer/Theodor W. Adorno, Elemente des Antisemitismus. Grenzen der Aufklärung, in: Helmut König 
Juden sind - verallgemeinert gesprochen - in der aktuellen Phase des Kapitalismus im deutschsprachigen Raum nicht strukturell, das heißt ökonomisch-institutionell ausgegrenzt ${ }^{68}$, wie dies etwa bei schwarzen Frauen in den USA der Fall ist. Allerdings dienen sie als Projektionsfläche eben jener ökonomisch-institutionellen Ausgrenzung, wie sie in Zuschreibungen wie „Spekulanten“, „Heuschrecken“ und „Machtelite“ deutlich werden. Die Verschränkung von Kapitalismus - bzw. dessen inhärenter Krisendynamik - und Antisemitismus wird insbesondere dadurch evident, dass der Antisemitismus seit dem ausgehenden 19. Jahrhundert immer dann an Brisanz gewann, „,wenn der Börsenkurs fiel““ ${ }^{69}$

Mit ihrer Arbeit zur „Intersektionalität von Ideologien“ legte Karin Stögner vor Kurzem ein Intersektionalitätskonzept vor, das den Antisemitismus als Analysekategorie zentral integriert. ${ }^{70}$ Stögner positioniert Antisemitismus dabei als eine Art Masterkategorie. Sie spricht von Antisemitismus als die, intersektionale Ideologie schlechthin, indem sie sichtbar macht, dass die Wirkmächtigkeit des Antisemitismus als verquere Welterklärung auch von seiner Durchdringung durch andere Ideologien wie Sexismus, Rassismus und Nationalismus herrührt“. ${ }^{71}$ Ein derartiger Anspruch einer Art „Masterkategorie“ findet sich allerdings auch in anderen Disziplinen, etwa in den Disability Studies, und führt nach Gudrun-Axeli Knapp zu keinen produktiven Auseinandersetzungen: „Eine bestimmte Auswahl der in Betracht zu ziehenden Verhältnisse in einer Konstellation [...] lässt sich nicht allgemein und normativ setzen, sondern nur materialiter und immer nur in je bestimmten Hinsichten". 72

Klaus Holz beschreibt Antisemitismus als ein „ideologisches Bewußtsein“"73 über die „Gesellschaft und sich selbst“, stets bedingt durch „die derzeitige Gesellschaftsform“. ${ }^{74}$ Der Antisemitismus erfüllt dabei die Funktion, mittels Projektionen ,sich die Welt zu erklären oder um Erklärungsmuster für die eigenen Marginalisierungserfahrungen zu finden, die es erlauben, sich nicht mit sich selbst auseinandersetzen zu müssen“. ${ }^{75}$ Derartige Marginalisierungserfahrungen werden zwar auf der individuellen Ebene erfahren, können in ihren Auswirkungen und (antisemitischen) Artikulationsformen aber

(Hrsg.), Elemente des Antisemitismus. Kommentare und Interpretationen zu einem Kapitel der Dialektik der Aufklärung von Max Horkheimer und Theodor W. Adorno, Weilerswist 2016, S. 21-52, hier S. 26.

68 Gelbin, Die jüdische Thematik.

69 Zit. n. Jan Weyand, Das Konzept der Kommunikationslatenz und der Fortschritt in der soziologischen Antisemitismusforschung, in: Jabrbuch für Antisemitismusforschung 26 (2017), S. 36-58, hier 39.

${ }^{70}$ Karin Stögner, Intersektionalität von Ideologien - Antisemitismus, Sexismus und das Verhältnis von Gesellschaft und Natur, in: Psychologie \& Gesellschaftskritik 41 (2017), Heft 2, S. 25-45.

${ }^{71}$ Ebd., S. 25.

${ }^{72}$ Gudrun-Axeli Knapp, Von Herkünften, Suchbewegungen und Sackgassen: Ein Abschlusskommentar, in: Sabine Hess/Nikola Langreiter/Elisabeth Timm (Hrsg.), Intersektionalität revisited. Empirische, theoretische und methodische Erkundungen, Bielefeld 2011, S. 249-272, hier S. 267.6

${ }^{73}$ Holz, Antisemitismus.

74 Ebd., S. 164.

75 Astrid Messerschmidt, Antisemitismuskritik im Kontext migrationsgesellschaftlicher und geschlechterreflektierter Bildung, in: Jabrbuch für Antisemitismusforschung 22 (2013), S. 99-122, hier S. 108. 
nur auf einer Makro-Ebene adäquat analysiert werden und letztlich zeigen „how these categories are created, how they exploit and not simply oppress, and why they intersect". ${ }^{76}$ In diesem Sinne ist auch die antisemitismuskritische Bildungsarbeit, die bei der eigenen Diskriminierungserfahrung ansetzt - wie sie unter anderem von Astrid Messerschmidt eingefordert wird ${ }^{77}$-, lediglich als eine Form der Symptombehandlung zu verstehen, da sie die gesellschaftlichen Verhältnisse, welche den modernen Antisemitismus hervorbringen, unangetastet lassen.

Auch gegenwärtige Formen der Rassifizierung sind Produkt zeitgenössischer Gesellschaftsformen. Volkhard Mosler bezeichnet Rassismus als „eine ,von oben“ konstruierte Ideologie, ein Beispiel dafür, dass, wie Marx und Engels es formulierten, die ,Gedanken der herrschenden Klasse [...] in jeder Epoche die herrschenden Gedanken sind““. ${ }^{78}$ Wie Silvia Federici in ihrem Buch „Caliban und die Hexe“ nachzeichnet, dient auch der Rassismus als bürgerliche Ideologie der Aufrechterhaltung der bestehenden Verhältnisse. ${ }^{79}$ Die Auffassung von intersektionalen Verhältnissen als Ideologien, wie von Stögner vorgeschlagen, eröffnet die Möglichkeit der Analyse und Kritik gesellschaftlicher Verhältnisse, die rassistische, sexistische und antisemitische Vorstellungen immer wieder auf das Neue aus sich selbst hervorbringt, und die zu deren Stabilisierung beständig beitragen. Gerade auch die Überkreuzungen dieser Weltbilder können auf diese Weise treffend erfasst werden.

\section{Zusammenfassung}

Dieser Beitrag ging der Frage nach, warum Antisemitismus bis heute lediglich eine marginale Rolle in den Debatten zur Intersektionalität spielt. Nach Said schreiben Forschende „,sowohl für eine Situation als auch in einer Situation“. ${ }^{80}$ Wie gezeigt wurde, sind zentrale Unterschiede zwischen den USA und dem deutschsprachigen Raum zu verzeichnen: vor allem hinsichtlich historischer Entwicklungen und (Dis)Kontinuitäten der gesellschaftlichen Verhältnisse, zu deren Kritik das Konzept der intersectionality/ Intersektionalität entwickelt wurde und in die dieses eingebettet war.

Ausgehend von Edward Saids Konzeption einer „traveling theory“ und der Annahme, dass ein ,zentraler Bezug auf ein in der Vergangenheit lokalisiertes Ereignis als bestimmend für die Identität ${ }^{\text {"81 }}$ wirkt, wurde nachgezeichnet, warum am Ursprungsort von intersectionality Antisemitismus kein zentraler Bestandteil der einschlägigen

${ }^{76}$ Salem, Intersectionality, S. 6.

${ }_{77}$ Messerschmidt, Antisemitismuskritik.

78 Volkhard Mosler, Rassismus im Wandel. Vom Sozialdarwinismus zum Kampf der Kulturen, in: theorie21 2 (2012),

S. 19-52, hier S. 20.

${ }^{79}$ Silvia Federici, Caliban und die Hexe. Frauen, der Körper und die ursprüngliche Akkumulation, Wien 2012.

80 Said, Theorien, S. 277.

81 Gelbin, Die jüdische Thematik, S. 94. 
Debatten war. Der historische Bezugspunkt am Entstehungsort des Konzeptes war die im Nachhall der Sklaverei begründete Bürgerrechtsbewegung und eine von der Studierendenbewegung mitgeprägte Frauenbewegung. Aus dem Konflikt mit diesen beiden Bewegungen begannen schwarze Frauen, sich selbständig zu organisieren und auf ihre spezifischen Erfahrungen und Benachteiligungen aufmerksam $\mathrm{zu}$ machen. Benachteiligungen, die sich auch juristisch bemerkbar machten, und die Kimberlé Crenshaw anhand von intersectionality in der bis heute dominierenden Trias race, class und gender ausarbeitete.

Entsprechend Saids Argumentation wurde auch der historische Kontext der neuen Umgebung skizziert. Im deutschsprachigen Raum charakterisierten die nationalsozialistische Vergangenheit und die Shoah die Auseinandersetzungen der 68erund der Frauenbewegung und führte paradoxerweise gerade deshalb lange Zeit zur Ausblendung von Antisemitismus als Unterdrückungsform und zu dessen fehlender Integration in das Analysekonzept Intersektionalität. 